\title{
Region of Attraction Comparison for Gradient Projection Anti-windup Compensated Systems
}

\author{
Justin Teo and Jonathan P. How
}

\begin{abstract}
The gradient projection anti-windup (GPAW) scheme was recently proposed for saturated multi-input-multioutput (MIMO) nonlinear systems driven by MIMO nonlinear controllers, a topic recognized as an open problem in a recent survey paper. Thus far, stability results for GPAW compensated systems are restricted to the simple case of a saturated first order linear time invariant (LTI) plant driven by a first order LTI controller. Here, we present a region of attraction (ROA) comparison result for general GPAW compensated regulatory systems. The ROA comparison result is demonstrated on a simple planar nonlinear system, which also highlights the limitations of existing state-of-the-art anti-windup results.
\end{abstract}

\section{INTRODUCTION}

The gradient projection anti-windup (GPAW) scheme was proposed in [1] for saturated multi-input-multi-output (MIMO) nonlinear systems driven by MIMO nonlinear controllers, a topic recognized as an open problem in the recent survey paper [2]. The GPAW scheme is a generalization of the well-known conditional integration method [3, p. 38], [4] to the case of MIMO nonlinear controllers [5, p. 52]. The construction of the GPAW compensated controller is detailed in [5, Chapter 2, pp. $33-70]$ along with some of its fundamental properties. It was shown in [6], [5, Theorem 2.5.3, p. 50] that the GPAW scheme achieves controller state-output consistency, a property unique to the GPAW scheme while being an implicit objective for the majority of anti-windup schemes [5, Remark 2.21, pp. $51-52]$.

The general anti-windup problem for nonlinear systems is presented in [5, Section 1.3, pp. 23 - 26]. Briefly, it states that given some nominal controller designed for the unconstrained system, nominal performance is recovered when no controls saturate, and that stability and performance of the anti-windup compensated system must be no worse than those of the saturated uncompensated system. ${ }^{1}$ One crucial difference with the problem considered in current antiwindup literature is that results relative to the uncompensated system are sought. More specifically, results which show that the stability and performance of the uncompensated system can only be maintained/improved with anti-windup compensation. While such requirements appear fundamental, it has not been specified in present anti-windup literature to the best of our knowledge. These requirements are further

J. Teo is with DSO National Laboratories, Singapore. (e-mail: justinteo@alum.mit.edu)

J. P. How is the Richard Cockburn Maclaurin Professor of Aeronautics and Astronautics at MIT. (e-mail: jhow@mit .edu)

${ }^{1}$ For regulatory systems, this requires the region of attraction (ROA) of the uncompensated system to be contained within the ROA of the anti-windup compensated system. motivated in [5, Section 3.7, pp. 94 - 96], where the superiority of such relative results are made apparent.

In this new framework, strong stability results have been obtained when restricted to the case of a saturated first order linear time invariant (LTI) plant driven by a first order LTI controller. In particular, the region of attraction (ROA) of the uncompensated system can only be maintained/enlarged by GPAW compensation [5, Proposition 3.5.13, p. 90], [7], [8]. In this paper, we present an ROA comparison result for MIMO nonlinear systems/controllers, which states conditions to ensure that the ROA (or ROA estimate) of the uncompensated system is never reduced by GPAW compensation. We construct a simple nonlinear planar system to demonstrate the application of this ROA comparison result. This same system is shown to elude current state-of-the-art anti-windup methods for LTI systems [2], [9], [10]; feedback linearizable nonlinear systems ${ }^{2}$ [11]; and Euler-Lagrange systems [12].

The rest of the paper is organized as follows. A general ROA comparison result is presented in Section II, followed by the main result in Section III. The example presented in Section IV is significant as it highlights the limitations of current state-of-the-art anti-windup results. The Appendix which follows the Conclusions summarizes the construction of the GPAW compensated controller.

We will adopt the following conventions. For any integer $i>0$, we define $\mathcal{I}_{i}:=\{1,2, \ldots, i\}$. Inequalities involving vectors are to be interpreted element-wise. The symbol $\wedge$ denotes logical conjunction.

\section{A General ROA Comparison Result}

Here, we present a general result that allows the size of the ROA of an autonomous system to be inferred from that of a related autonomous system. First, we recall some basic definitions. Consider the $n$-th order autonomous system

$$
\dot{x}=f(x),
$$

where for some domain $D \subset \mathbb{R}^{n}$, we assume the function $f: D \rightarrow \mathbb{R}^{n}$ satisfies some regularity conditions to ensure existence and uniqueness of solutions for all forward times. When $f$ is continuous, classical results based on Lipschitz continuity of $f$, e.g. [13, Section 3.1, pp. 88 - 95], are available to ensure existence and uniqueness of solutions for all (forward and reverse) times. If $f$ is discontinuous, ${ }^{3}$

\footnotetext{
${ }^{2}$ The majority of anti-windup schemes for nonlinear systems are constructed for feedback linearizable systems. See [5, Section 1.4.2, p. 27] for a more comprehensive list of references.

${ }^{3}$ The vector field of GPAW compensated systems will be discontinuous in general (see the switching conditions in (19) and [5, p. 76])
} 
regularity conditions are available to ensure existence and uniqueness of solutions for all forward times [14, §2.7, pp. 75 -86 , and $\S 2.10$, pp. $106-117]$. Let $\phi\left(t, x_{0}\right)$ be the unique solution of system (1) starting from $x_{0}$ at time $t=0$. A set $M$ is a positively invariant set for system (1) if the solution starting within $M$ stays in $M$ for all forward times, i.e. $x_{0} \in M \Rightarrow\left(\phi\left(t, x_{0}\right) \in M, \forall t \geq 0\right)$ [13, p. 127].

We assume that $x_{e q} \in D$ is an asymptotically stable equilibrium point for system (1). The ROA of the equilibrium $x_{e q}$ for system (1) is defined by [13, p. 314]

$$
R_{A}\left(x_{e q}\right):=\left\{\bar{x} \in \mathbb{R}^{n} \mid \phi(t, \bar{x}) \rightarrow x_{e q} \text { as } t \rightarrow \infty\right\} .
$$

In other words, the ROA is the set of all points such that the solution starting from any point within it converges to the equilibrium. Clearly, $R_{A}\left(x_{e q}\right)$ must contain the equilibrium $x_{e q}$. A set $\Omega \subset R_{A}\left(x_{e q}\right)$ is said to be an estimate of $R_{A}\left(x_{e q}\right)$ if every solution starting in $\Omega$ approaches $x_{e q}$ as $t \rightarrow \infty$ [13, p. 316], i.e. $x_{0} \in \Omega \Rightarrow \lim _{t \rightarrow \infty} \phi\left(t, x_{0}\right)=x_{e q}$. Clearly, a set is an estimate of $R_{A}\left(x_{e q}\right)$ if and only if it is a subset of $R_{A}\left(x_{e q}\right)$ (including the subset $R_{A}\left(x_{e q}\right)$ ). Numerous methods are available to estimate ROAs, e.g. [15], [16]. Our purpose is not to estimate ROAs, but, given an ROA estimate for a system, to find conditions for which the same ROA estimate is valid for a related system. Recall that a continuous function $\alpha:[0, a) \rightarrow[0, \infty)$ is said to belong to class $\mathcal{K}$ if it is strictly increasing and $\alpha(0)=0$ [13, Definition 4.2, p. 144].

An ROA estimate $\Omega \subset R_{A}\left(x_{e q}\right)$ is said to be associated with a Lyapunov function $V: \Omega \rightarrow \mathbb{R}$ for system (1) if

$$
\begin{gathered}
V\left(x_{e q}\right)=0, \quad V(\bar{x})>0, \quad \forall \bar{x} \in \Omega \backslash\left\{x_{e q}\right\}, \\
\dot{V}(\bar{x})=\frac{\partial V(\bar{x})}{\partial x} f(\bar{x}) \leq-\alpha\left(\left\|\bar{x}-x_{e q}\right\|\right), \quad \forall \bar{x} \in \Omega,
\end{gathered}
$$

for some class $\mathcal{K}$ function $\alpha:[0, a) \rightarrow[0, \infty)$, where $a:=$ $\sup _{\bar{x} \in \Omega}\left\|\bar{x}-x_{e q}\right\|$. In this case, we indicate the association by denoting such an estimate by $\Omega_{V}$. Note that numerous ROA estimation methods yield estimates that are associated with Lyapunov functions.

The next result gives sufficient conditions for an ROA estimate of a system associated with some Lyapunov function to be a valid ROA estimate for a related system, i.e. the estimate is contained within the ROA of this related system.

Lemma 1 ( [5, Lemma 4.3.1, p. 122]): Consider two $n$ th order autonomous systems

$$
\begin{aligned}
& \dot{x}=f_{1}(x), \\
& \dot{x}=f_{2}(x) .
\end{aligned}
$$

For some $D \subset \mathbb{R}^{n}$, assume $f_{1}: D \rightarrow \mathbb{R}^{n}$ and $f_{2}: D \rightarrow \mathbb{R}^{n}$ are such that solutions $\phi_{1}\left(t, x_{0}\right)$ and $\phi_{2}\left(t, x_{0}\right)$ to systems (3) and (4) respectively exist and are unique for all $x_{0} \in D$ and all $t \geq 0$. Assume $x_{e q} \in D$ is an asymptotically stable equilibrium point for both systems, and let $R_{A 1}\left(x_{e q}\right)$, $R_{A 2}\left(x_{e q}\right)$ be the ROAs of systems (3) and (4) respectively. Let $\Omega_{V} \subset R_{A 1}\left(x_{e q}\right)$ be an estimate of $R_{A 1}\left(x_{e q}\right)$ associated with a Lyapunov function $V: \Omega_{V} \rightarrow \mathbb{R}$ satisfying (2) (with $f_{1}, \Omega_{V}$ in place of $f, \Omega$ ). If $\Omega_{2}$ is a subset of $\Omega_{V}$ (possibly
$\Omega_{2}=\Omega_{V}$ ) and also a positively invariant set for system (4), and in addition,

$$
\frac{\partial V(\bar{x})}{\partial x} f_{2}(\bar{x}) \leq \frac{\partial V(\bar{x})}{\partial x} f_{1}(\bar{x}), \quad \forall \bar{x} \in \Omega_{2},
$$

then $\Omega_{2}$ is an estimate of $R_{A 2}\left(x_{e q}\right)$, i.e. $\Omega_{2} \subset R_{A 2}\left(x_{e q}\right)$.

Proof: The proof has some similarities with the proof of [17, Lemma 1]. We need to show that $\lim _{t \rightarrow \infty} \phi_{2}\left(t, x_{0}\right)=$ $x_{e q}$ for all $x_{0} \in \Omega_{2}$. Since $x_{e q}$ is an asymptotically stable equilibrium for system (4), $R_{A 2}\left(x_{e q}\right)$ is non-empty and necessarily contains $x_{e q}$, i.e. $x_{e q} \in R_{A 2}\left(x_{e q}\right) \neq \emptyset$. Since $R_{A 2}\left(x_{e q}\right)$ is open [13, Lemma 8.1, p. 314], it contains some sufficiently small neighborhood of $x_{e q}$. Hence there exists a sufficiently small $\epsilon>0$ such that $B_{\epsilon}:=\left\{\bar{x} \in \mathbb{R}^{n} \mid V(\bar{x}) \leq\right.$ $\epsilon\} \subset R_{A 2}\left(x_{e q}\right)$. Fix any such $\epsilon>0$.

From $\Omega_{2} \subset \Omega_{V},(5)$, and (2), we have for all $\bar{x} \in \Omega_{2}$,

$$
\frac{\partial V(\bar{x})}{\partial x} f_{2}(\bar{x}) \leq \frac{\partial V(\bar{x})}{\partial x} f_{1}(\bar{x}) \leq-\alpha\left(\left\|\bar{x}-x_{e q}\right\|\right) .
$$

Let $\partial B_{\epsilon}$ be the boundary of $B_{\epsilon}$, i.e. $\partial B_{\epsilon}=\left\{\bar{x} \in \mathbb{R}^{n}\right.$ $V(\bar{x})=\epsilon\}, \beta:=\min _{\bar{x} \in \partial B_{\epsilon}}\left\|\bar{x}-x_{e q}\right\|$, and $\delta:=\alpha(\beta)$. Clearly, we have $\beta>0$ and $\delta>0$ due to $\epsilon>0$ and (2). Then for all $\bar{x} \in \Omega_{2} \backslash B_{\epsilon}$, we have $\left\|\bar{x}-x_{e q}\right\| \geq \beta, \alpha\left(\left\|\bar{x}-x_{e q}\right\|\right) \geq$ $\alpha(\beta)=\delta$, and

$$
\frac{\partial V(\bar{x})}{\partial x} f_{2}(\bar{x}) \leq-\alpha\left(\left\|\bar{x}-x_{e q}\right\|\right) \leq-\delta .
$$

Let $x_{0} \in \Omega_{2}$. Since $\Omega_{2}$ is a positively invariant set for system (4), we have $\phi_{2}\left(t, x_{0}\right) \in \Omega_{2}$ for all $t \geq 0$. The Lyapunov function $V$ evaluated along $\phi_{2}\left(t, x_{0}\right)$ is given by

$V\left(\phi_{2}\left(t, x_{0}\right)\right)=V\left(x_{0}\right)+\int_{0}^{t} \frac{\partial V\left(\phi_{2}\left(\tau, x_{0}\right)\right)}{\partial x} f_{2}\left(\phi_{2}\left(\tau, x_{0}\right)\right) d \tau$,

for all $t \geq 0$. Clearly, $\phi_{2}\left(t, x_{0}\right)$ can remain in $\Omega_{2} \backslash B_{\epsilon}$ only for a finite amount of time. Otherwise, $\phi_{2}\left(t, x_{0}\right) \in \Omega_{2} \backslash B_{\epsilon}$ for all $t \geq 0$ and (6) imply

$V\left(\phi_{2}\left(t, x_{0}\right)\right) \leq V\left(x_{0}\right)-\delta \int_{0}^{t} d \tau=V\left(x_{0}\right)-\delta t, \quad \forall t \geq 0$,

which shows that $V\left(\phi_{2}\left(t, x_{0}\right)\right)<0$ for sufficiently large $t$, a contradiction to $V$ being positive definite. Hence $\phi_{2}\left(t, x_{0}\right)$ must enter $B_{\epsilon}$ at some finite time, and must approach $x_{e q}$ since $B_{\epsilon} \subset R_{A 2}\left(x_{e q}\right)$, i.e. $\lim _{t \rightarrow \infty} \phi_{2}\left(t, x_{0}\right)=x_{e q}$.

Remark 1: Note that the ROA estimate $\Omega_{V}$ in Lemma 1 need not be compact. Moreover, the conclusion $\Omega_{2} \subset$ $R_{A 2}\left(x_{e q}\right)$ shows that the (possibly unknown) ROA $R_{A 2}\left(x_{e q}\right)$ is at least as large as $\Omega_{2}$.

Lemma 1 will be specialized in the next section to GPAW compensated systems.

\section{ROA COMPARISON FOR GPAW COMPENSATED SYSTEMS}

For any well-posed anti-windup problem, the inputconstrained nominal system must be stable in some sense at least locally, e.g. locally asymptotically stable with respect to some equilibrium. When it is indeed locally asymptotically stable with respect to some equilibrium, the fundamental question is whether an anti-windup scheme can 
maintain/enlarge the ROA while achieving performance enhancements in the presence of control saturation.

Here, we present the main result which provides conditions under which the ROA of the regulatory GPAW compensated system is "lower bounded" by some ROA estimate of the uncompensated system. First, we describe the closed-loop systems. The open-loop plant is described by

$$
\begin{aligned}
& \dot{x}=f(x, \operatorname{sat}(u)), \quad x(0)=x_{0}, \\
& y=g(x, \operatorname{sat}(u)),
\end{aligned}
$$

where $x, x_{0} \in \mathbb{R}^{n}$ are its state and initial state, $y \in \mathbb{R}^{p}$ is the measurement, $u=\left[u_{1}, u_{2}, \ldots, u_{m}\right] \in \mathbb{R}^{m}$ is the plant input, and the saturation function sat: $\mathbb{R}^{m} \rightarrow \mathbb{R}^{m}$ is defined by

$$
\begin{gathered}
\operatorname{sat}(u)=\left[\rho_{1}\left(u_{1}\right), \rho_{2}\left(u_{2}\right), \ldots, \rho_{m}\left(u_{m}\right)\right]^{\mathrm{T}}, \\
\rho_{i}\left(u_{i}\right)=\max \left\{\min \left\{u_{i}, u_{\max , i}\right\}, u_{\min , i}\right\}, \quad \forall i \in \mathcal{I}_{m},
\end{gathered}
$$

for some $u_{\max , i}, u_{\min , i} \in \mathbb{R}$ satisfying $u_{\min , i}<u_{\max , i}$ for all $i \in \mathcal{I}_{m}$. The nominal controller is

$$
\begin{aligned}
& \dot{x}_{c}=f_{c}\left(x_{c}, y, r\right), \quad x_{c}(0)=x_{c 0}, \\
& u_{c}=g_{c}\left(x_{c}\right),
\end{aligned}
$$

where $x_{c}, x_{c 0} \in \mathbb{R}^{q}$ are its state and initial state, $u_{c} \in \mathbb{R}^{m}$ is the controller output, and $r:=r(t) \in \mathbb{R}^{n_{r}}$ is the instantaneous value of some exogenous input.

Making appropriate substitutions in (7) and (8), the nominal uncompensated system with $u=u_{c}$ can be written as

$$
\Sigma_{n}:\left\{\begin{aligned}
\dot{x} & =f\left(x, \operatorname{sat}\left(g_{c}\left(x_{c}\right)\right)\right), \\
\dot{x}_{c} & =f_{c}\left(x_{c}, g\left(x, \operatorname{sat}\left(g_{c}\left(x_{c}\right)\right)\right), r\right),
\end{aligned}\right.
$$

or $\Sigma_{n}: \dot{z}_{n}=f_{n}\left(z_{n}\right)$. The GPAW compensated system comprising the plant (7) and GPAW compensated controller (see (21) in Appendix) with $u=u_{g}$ is described by

$$
\Sigma_{g}:\left\{\begin{aligned}
\dot{x} & =f\left(x, \operatorname{sat}\left(g_{c}\left(x_{g}\right)\right)\right), \\
\dot{x}_{g} & =R_{\mathcal{I}^{*}} f_{c}\left(x_{g}, g\left(x, \operatorname{sat}\left(g_{c}\left(x_{g}\right)\right)\right), r\right),
\end{aligned}\right.
$$

where $R_{\mathcal{I}^{*}} f_{c}\left(x_{g}, y, r\right):=R_{\mathcal{I}^{*}}\left(x_{g}, y, r\right) f_{c}\left(x_{g}, y, r\right)$. Similarly, this can be written as $\Sigma_{g}: \dot{z}_{g}=f_{g}\left(z_{g}\right)$. In (9), $f_{n}: \mathbb{R}^{n+q} \rightarrow \mathbb{R}^{n+q}$ is the vector field of the nominal system with state $z_{n}:=\left(x, x_{c}\right)$, while in (10), $f_{g}: \mathbb{R}^{n+q} \rightarrow \mathbb{R}^{n+q}$ is the vector field of the GPAW compensated system with state $z_{g}:=\left(x, x_{g}\right)$.

Controller state-output consistency is a fundamental property of GPAW compensated controllers, stated as Theorem 3 in the Appendix. It implies that the unsaturated region

$$
\mathbb{R}^{n} \times K, \quad K:=\left\{\bar{x} \in \mathbb{R}^{q} \mid \operatorname{sat}\left(g_{c}(\bar{x})\right)=g_{c}(\bar{x})\right\},
$$

is a positively invariant set for the GPAW compensated system $\Sigma_{g}$ [5, Remark 2.18, p. 51]. When the controller state is initialized such that $x_{g}(0) \in K$, Theorem 3 shows that the GPAW compensated system (10) can be written as

$$
\Sigma_{g u}:\left\{\begin{aligned}
\dot{x} & =f\left(x, g_{c}\left(x_{g}\right)\right), \\
\dot{x}_{g} & =R_{\mathcal{I}^{*}} f_{c}\left(x_{g}, g\left(x, g_{c}\left(x_{g}\right)\right), r\right),
\end{aligned}\right.
$$

or $\Sigma_{g u}: \dot{z}_{g}=f_{g u}\left(z_{g}\right)$, where the saturation function $\operatorname{sat}(\cdot)$ has been eliminated. For this system, comparison would be made against the unconstrained system

$$
\Sigma_{u}:\left\{\begin{aligned}
\dot{x} & =f\left(x, g_{c}\left(x_{c}\right)\right), \\
\dot{x}_{c} & =f_{c}\left(x_{c}, g\left(x, g_{c}\left(x_{c}\right)\right), r\right),
\end{aligned}\right.
$$

or $\Sigma_{u}: \dot{z}_{u}=f_{u}\left(z_{u}\right)$ with state $z_{u}:=\left(x, x_{c}\right)$.

We assume that solutions of the nominal system $\Sigma_{n}$ and unconstrained system $\Sigma_{u}$ exist and are unique for all initial conditions and all times. This is a mild assumption as existence and uniqueness of solutions to the nominal and unconstrained systems are usually guaranteed in the design of the nominal controller even when not explicitly sought. For the GPAW compensated systems $\Sigma_{g}$ and $\Sigma_{g u}$, we assume that their solutions exist and are unique for all forward times when started within the unsaturated region ${ }^{4} \mathbb{R}^{n} \times K$. We restrict its existence to forward times since in general, the vector field of the GPAW compensated system will be discontinuous at least on the saturation constraint boundary $\mathbb{R}^{n} \times \partial K$ (see the switching conditions in (19) and [5, p. 76]). For brevity, this assumption on existence and uniqueness of solutions will not be repeated in the sequel.

The preceding systems (9), (10), (12), and (13), are regulatory (in contrast to tracking systems) when the controller reference $r$ is constant. ${ }^{5}$ We are interested in asymptotic stability, for which it is meaningful only if the constant $r$ induces some isolated equilibria. Which of the (possibly multiple) isolated equilibria is to be taken as reference should be apparent for the particular application. We assume that $r$ induces an isolated equilibrium point $z_{e q}:=\left(\tilde{x}, \tilde{x}_{c}\right) \in \mathbb{R}^{n+q}$ for the nominal system (9) satisfying

$$
\begin{gathered}
f\left(\tilde{x}, g_{c}\left(\tilde{x}_{c}\right)\right)=0, \\
f_{c}\left(\tilde{x}_{c}, g\left(\tilde{x}, g_{c}\left(\tilde{x}_{c}\right)\right), r\right)=0, \\
u_{\min }<g_{c}\left(\tilde{x}_{c}\right)<u_{\max },
\end{gathered}
$$

where $u_{\max }:=\left[u_{\max , 1}, \ldots, u_{\max , m}\right]^{\mathrm{T}}$ and $u_{\min }:=$ $\left[u_{\min , 1}, \ldots, u_{\min , m}\right]^{\mathrm{T}}$ are the vectors of saturation limits. Notice that the last condition of (14) implies that the equilibrium $z_{e q}$ lies in the interior of the unsaturated region $\mathbb{R}^{n} \times K$. It is clear that any isolated equilibrium within the interior of the unsaturated region for system (9) must also be an isolated equilibrium for systems (10), (12), and (13), since their vector fields coincide in $\mathbb{R}^{n} \times(K \backslash \partial K)$, i.e.

$f_{n}(\bar{z})=f_{g}(\bar{z})=f_{g u}(\bar{z})=f_{u}(\bar{z}), \quad \forall \bar{z} \in \mathbb{R}^{n} \times(K \backslash \partial K)$.

For this $r$, we assume that $z_{e q}$ is a locally asymptotically stable equilibrium for the nominal system (9), which is $a$ standard assumption in the anti-windup setting. The preceding relation and (14) implies that it is also a locally asymptotically stable equilibrium for systems (10), (12), and (13).

\footnotetext{
${ }^{4}$ The restriction to initial conditions within the unsaturated region $\mathbb{R}^{n} \times K$ is for simplicity, since this can usually be enforced.

${ }^{5}$ When $r$ is a constant, it can be taken as part of the description of the function $f_{c}$ in (9), (10), (12), and (13). Then we can write $f_{n}, f_{g}, f_{g u}$, and $f_{u}$, in (9), (10), (12), and (13) respectively, not as a function of $r$. These result in autonomous system descriptions.
} 
Let the ROAs of $z_{e q}$ for systems (9), (10), (12), and (13) be $R_{n}\left(z_{e q}\right), R_{g}\left(z_{e q}\right), R_{g u}\left(z_{e q}\right)$, and $R_{u}\left(z_{e q}\right)$, respectively. We are interested in establishing ROA containment results like [5, Proposition 3.5.13, p. 90], [8, Proposition 4], where the size of $R_{g}\left(z_{e q}\right)$ and $R_{g u}\left(z_{e q}\right)$ is to be inferred from that of $R_{n}\left(z_{e q}\right)$ and $R_{u}\left(z_{e q}\right)$ respectively. However, recognizing that the controller state can usually be initialized arbitrarily, and hence it is possible to ensure $x_{g}(0) \in K$, we are interested only in the size of $R_{g}\left(z_{e q}\right)$ and $R_{g u}\left(z_{e q}\right)$ within the unsaturated region, i.e. $R_{g}\left(z_{e q}\right) \cap\left(\mathbb{R}^{n} \times K\right)$ and $R_{g u}\left(z_{e q}\right) \cap\left(\mathbb{R}^{n} \times K\right)$. From the definitions of $f_{g}(10)$, $f_{g u}(12)$, and $K(11)$, we have

$$
f_{g}(\bar{z})=f_{g u}(\bar{z}), \quad \forall \bar{z} \in \mathbb{R}^{n} \times K,
$$

while Theorem 3 shows that $\mathbb{R}^{n} \times K$ is a positively invariant set for systems $\Sigma_{g}$ and $\Sigma_{g u}$. This implies

$$
R_{g}\left(z_{e q}\right) \cap\left(\mathbb{R}^{n} \times K\right)=R_{g u}\left(z_{e q}\right) \cap\left(\mathbb{R}^{n} \times K\right) .
$$

Then, with the initial controller state $x_{g}(0) \in K$ and the initial plant state $x(0)$, the solutions of the GPAW compensated systems $\Sigma_{g}$ and $\Sigma_{g u}$ will converge to $z_{e q}$ if $\left(x(0), x_{g}(0)\right) \in R_{g}\left(z_{e q}\right) \cap\left(\mathbb{R}^{n} \times K\right)$.

The following is the main ROA comparison result that provides sufficient conditions to assert that some ROA estimate $\Omega_{V}$ of the nominal system $\Sigma_{n}$ (or unconstrained system $\Sigma_{u}$ ) is also an ROA estimate of the GPAW compensated system $\Sigma_{g}\left(\right.$ or $\Sigma_{g u}$ ). An additional restriction (in contrast to Lemma 1) has been placed on the description of the ROA estimate $\Omega_{V}$, namely that it is a sublevel set of the associated Lyapunov function, ${ }^{6}$ i.e. $\Omega_{V}=\left\{\bar{z} \in \mathbb{R}^{n+q} \mid V(\bar{z}) \leq c\right\}$ for some $c>0$. Numerous ROA estimation methods yield estimates of this form, e.g. [15], [16], so that this restriction is well justified.

Theorem 2 (ROA Containment): Consider the nominal system $\Sigma_{n}$ (9) and GPAW compensated system $\Sigma_{g}(10)$. Assume that $z_{e q}=\left(\tilde{x}, \tilde{x}_{c}\right)$ is an asymptotically stable equilibrium for systems $\Sigma_{n}$ and $\Sigma_{g}$ that satisfies (14), and let $R_{n}\left(z_{e q}\right), R_{g}\left(z_{e q}\right)$ be the respective ROAs for $\Sigma_{n}$ and $\Sigma_{g}$. Let $\Omega_{V}=\left\{\bar{z} \in \mathbb{R}^{n+q} \mid V(\bar{z}) \leq c\right\} \subset R_{n}\left(z_{e q}\right)$ for some $c>0$ be an estimate of $R_{n}\left(z_{e q}\right)$ associated with a Lyapunov function $V: \mathbb{R}^{n+q} \rightarrow \mathbb{R}, z=\left(x, x_{c}\right) \mapsto V\left(x, x_{c}\right)=V(z)$, satisfying

$$
\begin{gathered}
V\left(z_{e q}\right)=0, \quad V(\bar{z})>0, \quad \forall \bar{z} \in \Omega_{V} \backslash\left\{z_{e q}\right\}, \\
\dot{V}(\bar{z})=\frac{\partial V(\bar{z})}{\partial z} f_{n}(\bar{z}) \leq-\alpha\left(\left\|\bar{z}-z_{e q}\right\|\right), \quad \forall \bar{z} \in \Omega_{V},
\end{gathered}
$$

for some class $\mathcal{K}$ function $\alpha$, where $f_{n}$ is the vector field of the nominal system $\Sigma_{n}(9)$. If there exists ${ }^{7}$ a $\Gamma=\Gamma^{\mathrm{T}}>0 \in$ $\mathbb{R}^{q \times q}$ such that for all $\left(\bar{x}, \bar{x}_{c}\right) \in \Omega_{V} \cap\left(\mathbb{R}^{n} \times \partial K\right)$,

$$
\frac{\partial V\left(\bar{x}, \bar{x}_{c}\right)}{\partial x_{c}}\left(I-R_{\mathcal{I}^{*}}\right) f_{c}\left(\bar{x}_{c}, g\left(\bar{x}, g_{c}\left(\bar{x}_{c}\right)\right), r\right) \geq 0,
$$

holds, then GPAW compensation with parameter $\Gamma$ yields system $\Sigma_{g}(10)$ whose ROA contains $\Omega_{V K}:=\Omega_{V} \cap\left(\mathbb{R}^{n} \times\right.$ $K)$, i.e. $\Omega_{V K} \subset R_{g}\left(z_{e q}\right)$.

\footnotetext{
${ }^{6}$ Observe that $\Omega_{V}$ having this form implies that it contains the equilibrium $z_{e q}$ in its interior.

${ }^{7}$ Recall that $R_{\mathcal{I}^{*}}$ is defined with parameter $\Gamma$ (see (22) in Appendix).
}

Remark 2: Observe that $f_{c}$ in (16) is independent of the saturation function sat $(\cdot)$, and it needs to hold only on the boundary $\mathbb{R}^{n} \times \partial K$ of the unsaturated region $\mathbb{R}^{n} \times K$.

Proof: We will be applying Lemma 1 with (3) and (4) representing $\Sigma_{n}$ (9) and $\Sigma_{g}$ (10) respectively. First, we establish the analogue of (5) with respect to the subset $\Omega_{V K}:=\left(\Omega_{V} \cap\left(\mathbb{R}^{n} \times K\right)\right) \subset \Omega_{V}$. Define $f(\bar{z}):=$ $f\left(\bar{x}, \operatorname{sat}\left(g_{c}\left(\bar{x}_{c}\right)\right)\right), f_{c}(\bar{z}):=f_{c}\left(\bar{x}_{c}, g\left(\bar{x}, \operatorname{sat}\left(g_{c}\left(\bar{x}_{c}\right)\right)\right), r\right)$, and observe that $\operatorname{sat}\left(g_{c}\left(\bar{x}_{c}\right)\right)=g_{c}\left(\bar{x}_{c}\right)$ for all $\bar{x}_{c} \in K$. Then using (9), (10), and (16), we have

$$
\begin{aligned}
\frac{\partial V(\bar{z})}{\partial z} f_{g}(\bar{z}) & =\frac{\partial V\left(\bar{x}, \bar{x}_{c}\right)}{\partial x} f(\bar{z})+\frac{\partial V\left(\bar{x}, \bar{x}_{c}\right)}{\partial x_{c}} R_{\mathcal{I}^{*}} f_{c}(\bar{z}), \\
& \leq \frac{\partial V\left(\bar{x}, \bar{x}_{c}\right)}{\partial x} f(\bar{z})+\frac{\partial V\left(\bar{x}, \bar{x}_{c}\right)}{\partial x_{c}} f_{c}(\bar{z}), \\
& =\frac{\partial V(\bar{z})}{\partial z} f_{n}(\bar{z}),
\end{aligned}
$$

for all $\bar{z} \in \Omega_{V} \cap\left(\mathbb{R}^{n} \times \partial K\right)$. For all $\bar{z}$ in the interior of the unsaturated region, i.e. $\bar{z} \in \mathbb{R}^{n} \times(K \backslash \partial K)$, no saturation constraints are active, so that $R_{\mathcal{I}^{*}}=I$ (see (22)) and hence $f_{g}(\bar{z})=f_{n}(\bar{z})$. Together, these yield

$$
\frac{\partial V(\bar{z})}{\partial z} f_{g}(\bar{z}) \leq \frac{\partial V(\bar{z})}{\partial z} f_{n}(\bar{z}), \quad \forall \bar{z} \in \Omega_{V K}
$$

which is analogous to (5).

Next, we show that $\Omega_{V K}$ is a positively invariant set for $\Sigma_{g}$. Since $\Omega_{V}=\left\{\bar{z} \in \mathbb{R}^{n+q} \mid V(\bar{z}) \leq c\right\}$, we can express $\Omega_{V K}=\Omega_{V} \cap\left(\mathbb{R}^{n} \times K\right)$ as $\Omega_{V K}=\left\{\bar{z} \in \mathbb{R}^{n} \times K \mid\right.$ $V(\bar{z}) \leq c\}$. Theorem 3 shows $\mathbb{R}^{n} \times K$ to be a positively invariant set for $\Sigma_{g}$, so that for any $z_{0} \in \Omega_{V K}$, the solution $\phi_{g}\left(t, z_{0}\right)$ of $\Sigma_{g}$ satisfy $\phi_{g}\left(t, z_{0}\right) \in \mathbb{R}^{n} \times K$ for all $t \geq 0$. Conditions (17) and (15) show that $\frac{\partial V(\bar{z})}{\partial z} f_{g}(\bar{z}) \leq 0$ for all $\bar{z} \in \Omega_{V K}$. Together, these show $\Omega_{V K}$ to be a positively invariant set for $\Sigma_{g}$.

By making the following identifications with quantities in Lemma 1,

$$
\begin{aligned}
& f_{1} \sim f_{n}, \quad R_{A 1}\left(x_{e q}\right) \sim R_{n}\left(z_{e q}\right), \quad x_{e q} \sim z_{e q}, \\
& f_{2} \sim f_{g}, \quad R_{A 2}\left(x_{e q}\right) \sim R_{g}\left(z_{e q}\right), \quad \Omega_{2} \sim \Omega_{V K},
\end{aligned}
$$

it can be verified that all its hypotheses are satisfied. Application of Lemma 1 then yields $\Omega_{V K} \subset R_{g}\left(z_{e q}\right)$.

Remark 3: From [13, Lemma 4.3, p. 145], we see that a class $\mathcal{K}$ function $\alpha$ exists to satisfy the second condition of (15) when $\dot{V}$ is continuous and negative definite with respect to the equilibrium $z_{e q}$ in $\Omega_{V}$, i.e. when $\dot{V}$ is continuous and

$$
\dot{V}\left(z_{e q}\right)=0, \quad \dot{V}(\bar{z})<0, \quad \forall \bar{z} \in \Omega_{V} \backslash\left\{z_{e q}\right\} .
$$

Clearly, $\dot{V}$ is continuous when $V$ is continuously differentiable and $f_{n}$ is continuous.

Remark 4: The main condition in Theorem 2 is (16), which as mentioned, has $f_{c}$ independent of the saturation function. It can be verified that the proof is valid with minor modifications $^{8}$ when $\Sigma_{n}, \Sigma_{g}, f_{n}, f_{g}, R_{n}\left(z_{e q}\right), R_{g}\left(z_{e q}\right)$,

\footnotetext{
${ }^{8}$ The only modifications needed are that the condition $\operatorname{sat}\left(g_{c}\left(\bar{x}_{c}\right)\right)=$ $g_{c}\left(\bar{x}_{c}\right)$ for all $\bar{x}_{c} \in K$ is not needed, and define $f(\bar{z}):=f\left(\bar{x}, g_{c}\left(\bar{x}_{c}\right)\right)$, $f_{c}(\bar{z}):=f_{c}\left(\bar{x}_{c}, g\left(\bar{x}, g_{c}\left(\bar{x}_{c}\right)\right), r\right)$ without the saturation function.
} 
are replaced by $\Sigma_{u}, \Sigma_{g u}, f_{u}, f_{g u}, R_{u}\left(z_{e q}\right), R_{g u}\left(z_{e q}\right)$, respectively.

The observation of Remark 4 means that the ROA of the GPAW compensated system can be lower bounded in size by two ways: comparing against an ROA estimate of the nominal (saturated) system, or an ROA estimate of the unconstrained system. When comparing against an ROA estimate of the nominal system, condition (16) is a sufficient condition for GPAW compensation to yield ROA improvements over the nominal system with respect to the estimate. ${ }^{9}$ Usually, the ROA of the unconstrained system will be at least as large as the ROA of the constrained nominal system. There may exist a $\Gamma$ satisfying (16), yet no $\Gamma$ such that the analogue of (16) can hold with respect to a larger ROA estimate for the unconstrained system. ROA comparison against the nominal system is a relative result, while comparisons against the ROA of the unconstrained system is in some sense an "absolute" result, prevalent in current anti-windup literature. As shown in [5, Section 3.7, pp. 94 - 96], such "absolute" results may give some confidence in the application of the anti-windup scheme, but may not reveal any advantages gained by its adoption.

Theorem 2 gives sufficient conditions for GPAW compensation to yield ROA improvements, without any indication as to the existence of $\Gamma$ satisfying (16), nor its choice. Moreover, the definition of $R_{\mathcal{I}^{*}}$ requires the solution of an optimization problem in general (see Appendix), on every point in $\Omega_{V} \cap\left(\mathbb{R}^{n} \times \partial K\right)$. In general, it would not be easy to find a $\Gamma$ satisfying (16). Next, we show that Theorem 2 can be applied at least to a simple nonlinear system.

\section{An Illustrative Nonlinear EXAmple}

Here, we apply Theorem 2 on a simple second order nonlinear system. This example also highlights the limitations of three classes of current state-of-the-art anti-windup results, in the sense that they cannot be applied to this system.

The following nonlinear system with a first order saturated linear plant and a first order nonlinear nominal controller is adapted from [13, Example 8.9, pp. 318 - 320]

$$
\Sigma_{n}:\left\{\begin{array}{l}
\dot{x}=-\operatorname{sat}(u), \\
\dot{u}=x+\left(x^{2}-1\right) u,
\end{array}\right.
$$

which corresponds to the constrained nominal system (9). The objective is to regulate the system state about the origin $z_{e q}=(0,0)$. We take $u_{\max }=1$ and $u_{\min }=-1$. Using closed-form expressions for the GPAW compensated controller (see [5, equation (A.5), p. 187]), we obtain the GPAW compensated system as

$$
\Sigma_{g}: \begin{cases}\dot{x}=-\operatorname{sat}(u), & \text { if } A_{\max }, \\ \dot{u}= \begin{cases}0, & \text { if } A_{\min } \\ 0, & \text { otherwise } \\ x+\left(x^{2}-1\right) u,\end{cases} \end{cases}
$$

\footnotetext{
${ }^{9}$ If the estimate is exact within the unsaturated region, then we have $\Omega_{V K}=\left(R_{n}\left(z_{e q}\right) \cap\left(\mathbb{R}^{n} \times K\right)\right) \subset R_{g}\left(z_{e q}\right)$.
}

where the controller switching conditions are

$$
\begin{gathered}
A_{\text {max }} \Leftrightarrow u \geq u_{\max } \wedge x+\left(x^{2}-1\right) u>0, \\
A_{\text {min }} \Leftrightarrow u \leq u_{\text {min }} \wedge x+\left(x^{2}-1\right) u<0 .
\end{gathered}
$$

Observe that the preceding has similarities with the wellknown conditional integration method [3, p. 38], [4]. This is expected because the GPAW scheme is an extension of the conditional integration method.

Since an ROA estimate for the associated unconstrained system (13) $\Sigma_{u}: \dot{z}=f_{u}(z)$ or

$$
\Sigma_{u}:\left\{\begin{array}{l}
\dot{x}=-u \\
\dot{u}=x+\left(x^{2}-1\right) u,
\end{array}\right.
$$

is readily available in [13, Example 8.9 , pp. 318 - 320], we will use it to demonstrate the application of Theorem 2 (see Remark 4). The conclusion yields a containment result for the ROA of the alternate form of the GPAW compensated system

$$
\Sigma_{g u}: \begin{cases}\dot{x}=-u, & \text { if } A_{\max }, \\ \dot{u}= \begin{cases}0, & \text { if } A_{\min }, \\ 0, & \text { otherwise. } \\ x+\left(x^{2}-1\right) u,\end{cases} \end{cases}
$$

Remark 5: The majority of anti-windup literature is focused on saturated LTI plants driven by LTI controllers [2], [9], [10]. Since the nominal controller (described by $\dot{u}=$ $\left.x+\left(x^{2}-1\right) u\right)$ is nonlinear, this class of methods do not apply. For nonlinear systems, the literature is dominated by methods for feedback linearizable plants driven by a feedback linearizing controller, e.g. [11]. While the unconstrained plant (described by $\dot{x}=-u$ ) is indeed feedback linearizable (in fact, it is linear), the nominal controller is not a feedback linearizing controller, which renders this class of methods inapplicable. As for the method of [12] for Euler-Lagrange systems, a fundamental requirement is that the unconstrained system must be globally asymptotically stable. As shown in [13, Example 8.9, pp. 318 - 320], the unconstrained system (18) is only locally asymptotically stable, so that the method of [12] also cannot be applied. In summary, this simple second order system eludes all these state-of-the-art anti-windup schemes. We will show however, that Theorem 2 applies, suggesting its generality.

As shown in [13, Example 8.9, pp. 318 - 320], a Lyapunov function for system $\Sigma_{u}$ (18) is

$$
V(\bar{z})=\bar{z}^{\mathrm{T}} P \bar{z}, \quad P=\left[\begin{array}{cc}
1.5 & -0.5 \\
-0.5 & 1
\end{array}\right],
$$

and an ROA estimate associated with $V$ is the sublevel set $\Omega_{V}=\left\{\bar{z} \in \mathbb{R}^{2} \mid V(\bar{z}) \leq c\right\}$ with $c=2.25$. This choice of $c=2.25$ ensures $\dot{V}(\bar{z})=\frac{\partial V(\bar{z})}{\partial z} f_{u}(\bar{z})$ is negative definite in $\Omega_{V}$. Since $V$ is continuously differentiable and $f_{u}$ is continuous, (15) holds (see Remark 3). The ROA estimate $\Omega_{V}$ is illustrated in Fig. 1 together with $R_{u}\left(z_{e q}\right)$ and $R_{g u}\left(z_{e q}\right)$, the true ROAs of the unconstrained system $\Sigma_{u}$ 
and GPAW compensated system $\Sigma_{g u}$ respectively. ${ }^{10}$ While not proven, Fig. 1 suggests that GPAW compensation may enlarge the ROA of the unconstrained system.

To apply Theorem 2, we need to verify (16). Note that because the controller is first order, it is independent of any GPAW parameter $\Gamma$ (see [5, Remark B.1, pp. $201-$ 202]). From (18) and (19), we see that $f_{c}\left(\bar{x}_{c}, g\left(\bar{x}, g_{c}\left(\bar{x}_{c}\right)\right), r\right)$ in (16) translates to $\bar{x}+\left(\bar{x}^{2}-1\right) \bar{x}_{c}$. Defining $\gamma\left(\bar{x}, \bar{x}_{c}\right):=$ $\frac{\partial V\left(\bar{x}, \bar{x}_{c}\right)}{\partial x_{c}}\left(I-R_{\mathcal{I}^{*}}\right) f_{c}\left(\bar{x}_{c}, g\left(\bar{x}, g_{c}\left(\bar{x}_{c}\right)\right), r\right)$, we have that for all $\left(\bar{x}, \bar{x}_{c}\right) \in \mathbb{R}^{2}$,

$$
\begin{aligned}
\gamma\left(\bar{x}, \bar{x}_{c}\right) & =\left(2 \bar{x}_{c}-\bar{x}\right)\left(I-R_{\mathcal{I}^{*}}\right) f_{c}\left(\bar{x}_{c}, g\left(\bar{x}, g_{c}\left(\bar{x}_{c}\right)\right), r\right), \\
& = \begin{cases}\left(2 \bar{x}_{c}-\bar{x}\right)\left(\bar{x}+\left(\bar{x}^{2}-1\right) \bar{x}_{c}\right), & \text { if } A_{\max 1}, \\
\left(2 \bar{x}_{c}-\bar{x}\right)\left(\bar{x}+\left(\bar{x}^{2}-1\right) \bar{x}_{c}\right), & \text { if } A_{\min 1}, \\
0, & \text { otherwise }\end{cases}
\end{aligned}
$$

where $\quad A_{\max 1} \Leftrightarrow \bar{x}_{c} \geq u_{\max } \wedge \bar{x}+\left(\bar{x}^{2}-1\right) \bar{x}_{c}>0$,

$$
A_{\min 1} \Leftrightarrow \bar{x}_{c} \leq u_{\min } \wedge \bar{x}+\left(\bar{x}^{2}-1\right) \bar{x}_{c}<0 .
$$

For any $\left(\bar{x}, \bar{x}_{c}\right) \in \mathbb{R} \times \partial K=\mathbb{R} \times\left\{u_{\min }, u_{\max }\right\}$, we have from the preceding,

$\gamma\left(\bar{x}, \bar{x}_{c}\right)= \begin{cases}\left(2 u_{\max }-\bar{x}\right)\left(\bar{x}+\left(\bar{x}^{2}-1\right) u_{\max }\right), & \text { if } A_{\max 2}, \\ \left(2 u_{\min }-\bar{x}\right)\left(\bar{x}+\left(\bar{x}^{2}-1\right) u_{\min }\right), & \text { if } A_{\min 2}, \\ 0, & \text { otherwise }\end{cases}$

where $\quad A_{\max 2} \Leftrightarrow \bar{x}_{c}=u_{\max } \wedge \bar{x}+\left(\bar{x}^{2}-1\right) u_{\max }>0$,

$$
A_{\min 2} \Leftrightarrow \bar{x}_{c}=u_{\min } \wedge \bar{x}+\left(\bar{x}^{2}-1\right) u_{\min }<0 .
$$

By inspection of the preceding, we see that $\gamma\left(\bar{x}, \bar{x}_{c}\right) \geq 0$ for all $\left(\bar{x}, \bar{x}_{c}\right)=\left(\bar{x}, u_{\max }\right)$ when $\bar{x} \leq 2 u_{\max }=2$, and all $\left(\bar{x}, \bar{x}_{c}\right)=\left(\bar{x}, u_{\min }\right)$ when $\bar{x} \geq 2 u_{\min }=-2$. In other words, we have

$$
\begin{gathered}
\gamma\left(\bar{x}, \bar{x}_{c}\right) \geq 0, \quad \forall\left(\bar{x}, \bar{x}_{c}\right) \in X_{\gamma} \subset \mathbb{R} \times \partial K, \\
X_{\gamma}=\left((-\infty, 2] \times\left\{u_{\max }\right\}\right) \cup\left([-2, \infty) \times\left\{u_{\min }\right\}\right) .
\end{gathered}
$$

Condition (16) requires $\gamma\left(\bar{x}, \bar{x}_{c}\right) \geq 0$ for all $\left(\bar{x}, \bar{x}_{c}\right) \in$ $\Omega_{V} \cap(\mathbb{R} \times \partial K)$. Using the definition of $\Omega_{V}\left(\Omega_{V}=\{\bar{z} \in\right.$ $\left.\mathbb{R}^{2} \mid V(\bar{z}) \leq c\right\}$ for $\left.c=2.25\right)$, it can be verified that

$$
\Omega_{V} \cap(\mathbb{R} \times \partial K)=\left(\beta_{+} \times\left\{u_{\max }\right\}\right) \cup\left(\beta_{-} \times\left\{u_{\min }\right\}\right),
$$

where $\beta_{+}:=\left[\frac{1}{3}-\sqrt{\frac{17}{18}}, \frac{1}{3}+\sqrt{\frac{17}{18}}\right]=[-0.638,1.305]$ and $\beta_{-}:=\left[-\frac{1}{3}-\sqrt{\frac{17}{18}},-\frac{1}{3}+\sqrt{\frac{17}{18}}\right]=[-1.305,0.638]$. Since $\beta_{+} \subset$ $(-\infty, 2]$ and $\beta_{-} \subset[-2, \infty)$, we see from (20) that $\gamma\left(\bar{x}, \bar{x}_{c}\right) \geq 0$ for all $\left(\bar{x}, \bar{x}_{c}\right) \in\left(\beta_{+} \times\left\{u_{\max }\right\}\right) \cup\left(\beta_{-} \times\right.$ $\left.\left\{u_{\min }\right\}\right)=\Omega_{V} \cap(\mathbb{R} \times \partial K)$, which shows that (16) holds.

All hypotheses of Theorem 2 are satisfied, and we conclude from its application that the ROA of system $\Sigma_{g u}$ contains $\Omega_{V} \cap(\mathbb{R} \times K)$. Two sets of solutions, one starting from $z_{0}=(-1.2,-0.7) \in\left(\Omega_{V} \cap(\mathbb{R} \times K)\right) \subset R_{u}\left(z_{e q}\right) \subset$ $R_{g u}\left(z_{e q}\right)$ and another starting from $z_{0}=(2.8,0) \in$ $R_{g u}\left(z_{e q}\right) \backslash R_{u}\left(z_{e q}\right)$ are shown in Figs. 1 and 2.

\footnotetext{
${ }^{10}$ The ROAs $R_{u}\left(z_{e q}\right)$ and $R_{g u}\left(z_{e q}\right)$ are found numerically by a trial and error process together with backward-in-time simulations.
}

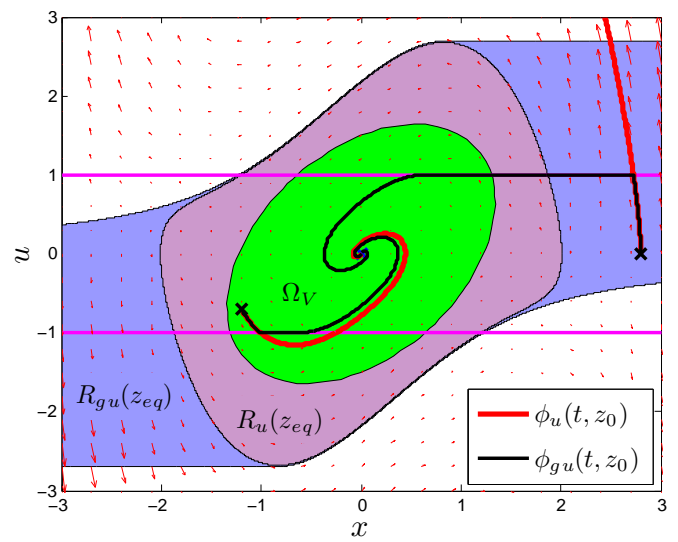

Fig. 1. ROA estimate $\Omega_{V}$ of a planar nonlinear system together with $R_{u}\left(z_{e q}\right)$ and $R_{g u}\left(z_{e q}\right)$, the true ROAs of the unconstrained system $\Sigma_{u}$ and GPAW compensated system $\Sigma_{g u}$ respectively.

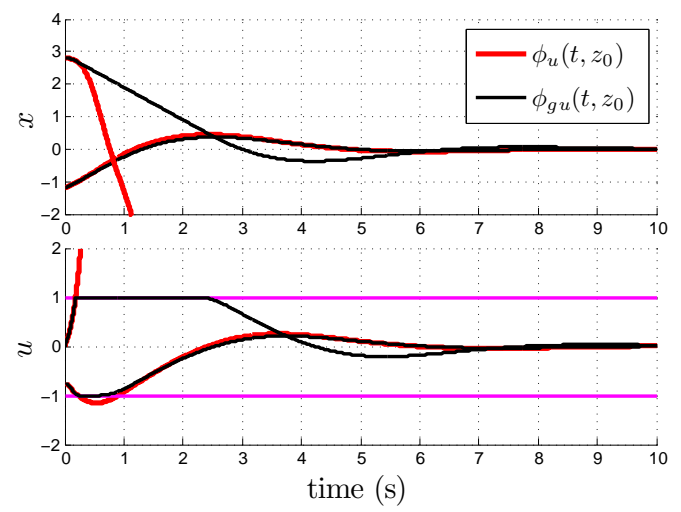

Fig. 2. Solutions of unconstrained and GPAW compensated systems.

\section{CONCLUSIONS}

In this paper, we extended stability results for gradient projection anti-windup (GPAW) compensated systems to saturated multi-input-multi-output (MIMO) nonlinear plants driven by MIMO nonlinear controllers. The fundamental question for anti-windup schemes is whether their application can improve performance without sacrificing stability. To the best of our knowledge, this question has not been explicitly addressed in the literature. For regulatory systems, this requires the region of attraction (ROA) of the uncompensated system to be contained within that of the antiwindup compensated system. The main result is a first step in this direction, which gives sufficient conditions for an ROA estimate of the uncompensated system to be a valid ROA estimate for the corresponding GPAW compensated system. A second order nonlinear example not only demonstrates the application of the main result, but highlights the limitations of existing state-of-the-art anti-windup results. Future work includes the search for qualitatively similar but less conservative ROA containment results.

\section{APPENDIX}

\section{Construction of GPAW COMPENSATEd CONTROLleR}

The construction of the GPAW compensated controller is detailed in [5, Chapter 2, pp. 33 - 70] (and also in [1], [6][8], [18]), which we summarize here. Due to lack of space, we direct the interested reader to these sources for details. 
Consider a nominal controller (8) that has been designed to achieve some nominal stability and performance. ${ }^{11}$ Application of GPAW compensation on the nominal controller (8) yields the GPAW compensated controller

$$
\begin{aligned}
& \dot{x}_{g}=R_{\mathcal{I}^{*}}\left(x_{g}, y, r\right) f_{c}\left(x_{g}, y, r\right), \quad x_{g}(0)=x_{c 0}, \\
& u_{g}=g_{c}\left(x_{g}\right),
\end{aligned}
$$

where $x_{g} \in \mathbb{R}^{q}$ is the state, $u_{g} \in \mathbb{R}^{m}$ is the output, $\left(y, r, f_{c}, g_{c}\right)$ remains unaltered as in (8), and the state-andinput dependent $q \times q$ matrix $R_{\mathcal{I}^{*}}\left(x_{g}, y, r\right)$ remains to be defined.

Define the $2 m$ saturation constraint functions $h_{i}$ by

$$
\begin{aligned}
h_{i}\left(x_{g}\right) & =g_{c i}\left(x_{g}\right)-u_{\max , i}, \quad \forall i \in \mathcal{I}_{m}, \\
h_{i+m}\left(x_{g}\right) & =-g_{c i}\left(x_{g}\right)+u_{\min , i},
\end{aligned}
$$

where $g_{c i}$ for $i \in \mathcal{I}_{m}$ are the elements of the controller output function $g_{c}=\left[g_{c 1}, g_{c 2}, \ldots, g_{c m}\right]^{\mathrm{T}}$ in (21). Assuming differentiability of $g_{c}$, these constraint functions have gradients

$$
\nabla h_{i}\left(x_{g}\right)=-\nabla h_{i+m}\left(x_{g}\right)=\nabla g_{c i}\left(x_{g}\right), \quad \forall i \in \mathcal{I}_{m} .
$$

For any index set $\mathcal{I} \subset \mathcal{I}_{2 m}$, define the $q \times \max \{|\mathcal{I}|, 1\}$ matrix $N_{\mathcal{I}}\left(x_{g}\right)= \begin{cases}{\left[\nabla h_{\sigma_{\mathcal{I}}(1)}\left(x_{g}\right), \ldots, \nabla h_{\sigma_{\mathcal{I}}(|\mathcal{I}|)}\left(x_{g}\right)\right],} & \text { if } \mathcal{I} \neq \emptyset, \\ 0, & \text { otherwise, }\end{cases}$ where $\sigma_{\mathcal{I}}:\{1,2, \ldots,|\mathcal{I}|\} \rightarrow \mathcal{I}$ is a chosen (non-unique) bijection that assigns an integer in $\mathcal{I}$ to each integer in $\{1,2, \ldots,|\mathcal{I}|\}$. See $[5$, Remark 2.5, p. 39$]$ for a more detailed description of the bijection $\sigma_{\mathcal{I}}$ and an example.

For any index set $\mathcal{I} \subset \mathcal{I}_{2 m}$ such that $\operatorname{rank}\left(N_{\mathcal{I}}\left(x_{g}\right)\right)=|\mathcal{I}|$, define the projection matrix $R_{\mathcal{I}}: \mathbb{R}^{q} \rightarrow \mathbb{R}^{q \times q}$

$$
\begin{gathered}
R_{\mathcal{I}}\left(x_{g}\right)=I-\tilde{S}_{\mathcal{I}}\left(x_{g}\right), \\
\tilde{S}_{\mathcal{I}}\left(x_{g}\right)= \begin{cases}\Gamma N_{\mathcal{I}}\left(N_{\mathcal{I}}^{\mathrm{T}} \Gamma N_{\mathcal{I}}\right)^{-1} N_{\mathcal{I}}^{\mathrm{T}}\left(x_{g}\right), & \text { if } \mathcal{I} \neq \emptyset, \\
0, & \text { otherwise, }\end{cases}
\end{gathered}
$$

where $\Gamma \in \mathbb{R}^{q \times q}$ is the single GPAW parameter, chosen to be symmetric positive definite. As shown in [5, Remark B.1, pp. 201 - 202], first order GPAW compensated controllers are fully defined independent of any parameters.

Define the index set of active saturation constraints $\mathcal{I}_{\text {sat }}$, and the candidate solution set $\mathcal{J}$

$$
\begin{aligned}
\mathcal{I}_{\text {sat }} & :=\mathcal{I}_{\text {sat }}\left(x_{g}\right)=\left\{i \in \mathcal{I}_{2 m} \mid h_{i}\left(x_{g}\right) \geq 0\right\}, \\
\mathcal{J} & :=\mathcal{J}\left(x_{g}\right)=\left\{\mathcal{I} \subset \mathcal{I}_{\text {sat }}|| \mathcal{I} \mid \leq q\right\}
\end{aligned}
$$

For any fixed $\left(x_{g}, y, r\right)$, let $\mathcal{I}^{*}$ be a solution to the following combinatorial optimization subproblem ${ }^{12}$

$$
\begin{aligned}
& \max _{\mathcal{I} \in \mathcal{J}} f_{c}^{\mathrm{T}}\left(x_{g}, y, r\right) \Gamma^{-1} R_{\mathcal{I}}\left(x_{g}\right) f_{c}\left(x_{g}, y, r\right), \\
& \text { subject to } \quad \operatorname{rank}\left(N_{\mathcal{I}}\left(x_{g}\right)\right)=|\mathcal{I}| \text {, } \\
& N_{\mathcal{I}_{s a t}}^{\mathrm{T}}\left(x_{g}\right) R_{\mathcal{I}}\left(x_{g}\right) f_{c}\left(x_{g}, y, r\right) \leq 0 .
\end{aligned}
$$

Then the GPAW compensated controller is defined by (21) with $R_{\mathcal{I}^{*}}\left(x_{g}, y, r\right)$ defined by (22) and $\mathcal{I}^{*}$. As shown in [5,

\footnotetext{
${ }^{11}$ As shown in [5, Section 2.6, pp. 53 - 55], more general controllers can be approximated arbitrarily well to have the form of (8).

${ }^{12}$ The existence of an optimal solution $\mathcal{I}^{*}$ to subproblem (23) is assured by [5, Proposition 2.5.1, p. 49].
}

Remark 2.17 , p. 50], GPAW compensation is active only when some controls saturate. Controller state-output consistency is a fundamental property of GPAW compensated controllers, stated below.

Theorem 3 ( [5, Theorem 2.5.3, p. 50]): Consider the GPAW compensated controller defined by (21), (22), and a solution $\mathcal{I}^{*}$ to subproblem (23). If there exists a $T \in \mathbb{R}$ such that $\operatorname{sat}\left(u_{g}(T)\right)=u_{g}(T)$, then $\operatorname{sat}\left(u_{g}(t)\right)=u_{g}(t)$ holds for all $t \geq T$.

Proof: See [5, pp. $50-51]$.

The preceding is the basic construction of the GPAW compensated controller. See [5, pp. 111 - 115, pp. 185 - 204] for alternate equivalent realizations.

\section{REFERENCES}

[1] J. Teo and J. P. How, "Anti-windup compensation for nonlinear systems via gradient projection: Application to adaptive control," in Proc. 48th IEEE Conf. Decision and Control \& 28th Chinese Control Conf., Shanghai, China, Dec. 2009, pp. $6910-6916$.

[2] S. Tarbouriech and M. Turner, "Anti-windup design: an overview of some recent advances and open problems," IET Control Theory Appl., vol. 3, no. 1, pp. 1 - 19, Jan. 2009.

[3] A. Visioli, Practical PID Control, ser. Adv. Ind. Control. London, United Kingdom: Springer, 2006.

[4] H. A. Fertik and C. W. Ross, "Direct digital control algorithm with anti-windup feature," ISA Trans., vol. 6, no. 4, pp. 317 - 328, 1967.

[5] C. S. J. Teo, "Gradient projection anti-windup scheme," Ph.D. dissertation, Massachusetts Institute of Technology, Cambridge, MA, Feb. 2011. [Online]. Available: http://hdl.handle.net/1721.1/63043

[6] J. Teo and J. P. How, "Geometric properties of gradient projection anti-windup compensated systems," in Proc. American Control Conf., Baltimore, MD, Jun./Jul. 2010, pp. 5973 - 5978.

[7] — " "Analysis of gradient projection anti-windup scheme," in Proc. American Control Conf., Baltimore, MD, Jun./Jul. 2010, pp. 5966 5972.

[8] —, "Gradient projection anti-windup scheme on constrained planar LTI systems," MIT, Cambridge, MA, Tech. Rep. ACL1001, Mar. 2010, Aerosp. Controls Lab. [Online]. Available: http://hdl.handle.net/1721.1/52600

[9] M. V. Kothare, P. J. Campo, M. Morari, and C. N. Nett, "A unified framework for the study of anti-windup designs," Automatica, vol. 30 , no. 12, pp. 1869 - 1883, Dec. 1994.

[10] C. Edwards and I. Postlethwaite, "Anti-windup and bumpless-transfer schemes," Automatica, vol. 34, no. 2, pp. 199 - 210, Feb. 1998.

[11] S.-S. Yoon, J.-K. Park, and T.-W. Yoon, "Dynamic anti-windup scheme for feedback linearizable nonlinear control systems with saturating inputs," Automatica, vol. 44, no. 12, pp. 3176 - 3180, Dec. 2008.

[12] F. Morabito, A. R. Teel, and L. Zaccarian, "Nonlinear antiwindup applied to Euler-Lagrange systems," IEEE Trans. Robot. Autom., vol. 20, no. 3, pp. 526 - 537, Jun. 2004.

[13] H. K. Khalil, Nonlinear Systems, 3rd ed. Upper Saddle River, NJ: Prentice Hall, 2002.

[14] A. F. Filippov, Differential Equations with Discontinuous Righthand Sides, ser. Math. Appl. Dordrecht, Netherlands: Kluwer Academic Publishers, 1988.

[15] W. Tan and A. Packard, "Stability region analysis using polynomial and composite polynomial Lyapunov functions and sum-of-squares programming," IEEE Trans. Autom. Control, vol. 53, no. 2, pp. $565-$ 571, Mar. 2008.

[16] A. I. Zečević and D. D. Šiljak, "Estimating the region of attraction for large-scale systems with uncertainties," Automatica, vol. 46, no. 2 , pp. 445 - 451, Feb. 2010.

[17] H. Fang and Z. Lin, "Stability analysis for linear systems under state constraints," IEEE Trans. Autom. Control, vol. 49, no. 6, pp. 950 955, Jun. 2004

[18] J. Teo and J. P. How, "Corrections to 'geometric properties of gradient projection anti-windup compensated systems'," MIT, Cambridge, MA, Tech. Rep. ACL10-02, Jun. 2010, Aerosp. Controls Lab. [Online]. Available: http://hdl.handle.net/1721.1/56001 